kann“, kommentierte Hans-Jörg Senn, St. Gallen, Schweiz, die Daten im Rahmen einer ESMO-Pressekonferenz. „Sie machen lieber die Gene und die Gesellschaft dafür verantwortlich, wenn sie Krebs bekommen."

Nach Senn besteht daher eine wesentliche gesundheitspolitische Aufgabe für die Zukunft darin, das Bewusstsein für die große Bedeutung der primären Krebsprävention zu erhöhen. Senn: „Wenn wir nicht erfolgreicher dabei werden, die Inzidenz der verbreiteten Krebsformen - wie z. B. gastrointestinaler Tumoren oder Brustkrebs - signifikant zu reduzieren, werden wir in unserer älter werdenden Gesellschaft mit dramatisch ansteigenden finanziellen Aufwendungen für ständig mehr Krebsbehandlung konfrontiert sein. Abgesehen von den vorhersagbaren Arbeitskraftverlusten und der enormen Belastung durch menschliches Leid.“

\section{Krebsbezogene Gesundheitsausgaben:}

Deutschland vorne

In Europa summieren sich direkte und indirekte Gesundheitsausgaben für die Behandlung von Tumorerkrankungen auf rund 120 Milliarden Euro pro Jahr. Zu diesem Ergebnis kommt die Arbeitsgruppe um Ramon Luengo-Fernandez, Oxford, Großbritannien [Luengo-Fernandez R et al. Ann Oncol. 2012;23(Suppl 9):Abstr. 1415 PD_PR]. Sie analysierten Daten der WHO, der OECD und der nationalen Gesundheitsministerien.

$36 \%$ der 120 Milliarden Euro entstanden durch Behandlungskosten. Einkommensausfälle wegen vorzeitiger Sterblichkeit und Morbidität machten 36 bzw. $8 \%$ der Kosten aus, unbezahlte Pflege summierte sich auf $20 \%$. Bezogen auf die jährlichen Pro-Kopf-Ausgaben für Krebs gibt Deutschland mit 165 Euro fünfmal so viel aus wie Litauen mit 32 Euro, dem Land mit den niedrigsten jährlichen Pro-Kopf-Ausgaben.

Brustkrebs war mit 6 Milliarden Euro (13\%) für die höchsten krebsbezogenen Gesundheitsausgaben in der EU verantwortlich. Lungenkrebs verursachte mit 19 Milliarden Euro (16\%) die höchsten Gesamtausgaben, gefolgt von kolorektalen Tumoren

\footnotetext{
kurz notiert

„Imagepflege“: Krebs wird viel zu negativ dargestellt

Die Diagnose Krebs ist für die Betroffenen ein Schock. Dass die Prognose vieler Krebserkrankungen heute weitaus besser ist als noch vor einem Jahrzehnt spiegelt sich in den Medien kaum wieder. Interessant ist dazu eine italienische Untersuchung, in der 82 Spielfilme analysiert wurden [Rosti G. et al. Ann Oncol. 2012;23(Suppl 9):Abstr. 1408]. Insgesamt erkrankten In diesen Filmen 75 Personen an Krebs (40 Frauen, 35 Männer), 46 davon verstarben. Am häufigsten waren es Lymphome, Leukämien oder Gehirntumoren. Als Therapien wurden vor allem Chemotherapie und Schmerztherapie genannt. Luciano De Fiore, Rom, Italien, kommentierte die Ergebnisse der Arbeit folgendermaßen: „Obwohl Brustkrebs einen starken Einfluss auf Frauen hat, ist er in

Spielfilmen kaum repräsentiert, stattdessen dominieren relativ seltene Krebsformen wir Leukämie, Lymphome und Gehirntumoren“ und weiter: „Die Vorstellungen und Konzepte von Krebs, wie sie in Filmen dargestellt werden, hinken hinter den heutigen therapeutischen Möglichkeiten her. Im Kino überlebt der Krebspatient nur sehr selten aufgrund der Therapie, im wirklichen Leben ist das glücklicher Weise in sehr vielen Fällen nicht mehr so." Seiner Ansicht nach wäre es wünschenswert, dass Filme zum Thema Krebs eine positive Auswirkung haben, z. B. indem sie Bewusstsein dafür schaffen, wie bedeutsam das Thema Krebs ist und welche neuen Therapien verfügbar sind. Doris Berger
}

n(11\%), Brust- (10\%) und Prastatakrebs (5\%). Als ein Motiv für die Studie führte Luengo-Fernandez an, die Ausgaben für verschiedene Krankheiten in der Europäischen Union vergleichbar machen zu wollen, um damit Entscheidungsträgern Hinweise für Prioritäten bei der Forschungsförderung geben zu können.

\section{Medikamenteninteraktionen}

\section{bei oraler Chemotherapie mehr beachten}

Bei fast jedem zweiten Krebspatienten, der ein orales Chemotherapeutikum erhält, tritt zumindest eine Wechselwirkung zwischen diesem und einem anderen Medikament auf, das der Patient einnimmt. Von fast 900 untersuchten Personen beobachtete die Arbeitsgruppe um Roelof van Leeuwen, Rotterdamm, Niederlande, bei 426 Studienteilnehmern (46\%) insgesamt 1.359 Wechselwirkungen, bei 143 (16\%) wurden diese als stark eingestuft [van Leeuwen RW et al. Ann Oncol. 2012;23(Suppl 9):Abstr. 1402P]. Am häufigsten traten Wechselwirkungen mit Opioid-Analgetika und Cumarin-Derivaten zur Gerinnungshemmung auf. Beobachtete Symptome betrafen das zentrale Nervensystem, den Gastrointestinaltrakt aber auch kardiologische Prozesse wie QTZeit-Verlängerungen im Elektrokardiogramm (EKG) kamen vor. Es gilt zu bedenken, dass neben akuten unerwünschten Wirkungen in ernsten Fällen Wechselwirkungen die Chemotherapie vollständig inaktivieren können oder der Patient durch eine auftretende Wirkungsverstärkung akut gefährdet wird. Als Beispiel nannte van Leeuwen die Wechselwirkung von Antidepressiva mit der Hormontherapie gegen Brustkrebs. Eine Reihe von Antidepressiva könne die Wirksamkeit von Tamoxifen so weit reduzieren, dass u. U. die antihormonelle Behandlung wirkungslos werde. Daher fordert er ein Computer-basiertes System. Darin sollen alle vom Patienten eingenommenen Medikamente erfasst werden und dass System sollte bei einem Risiko möglicher und gefährlicher Interaktionen Alarm schlagen.

\section{Sexuelle und soziale Probleme thematisieren}

In Fragebögen zur Lebensqualität werden Fatigue, Schmerzen Übelkeit und Erbrechen als die Symtome angegeben, die das Befinden des Patienten am stärksten beeinflussen. Gerade diese $\mathrm{Ne}$ benwirkungen einer Tumortherapie lassen sich allerdings mit adäquater Supportivtherapie heute gut behandeln. Müssen andere Aspekte mehr berücksichtigt werden? Felix K. Tauchert, Frankfurt/Main, und Kollegen befragten daher 1.300 Patienten, welche Symptome sie am meisten belasten und inwieweit diese im ArztPatienten-Gespräch thematisiert werden [Tauchert FK et al. Ann Oncol. 2012;23(Suppl 9):Abstr. 1413TiP]. Das Ergebnis: Schwäche, Müdigkeit, Konzentrationsstörungen, Zukunftsängste sowie therapiebedingter Haarausfall wurden als sehr belastend empfunden. Viele der Patienten beklagten darüber hinaus die fehlende Ansprache folgender Aspekte im Rahmen des Arzt-PatientenGespräches: Einschränkungen des Sexuallebens, soziale Probleme, Depressionen, Angstzustände, Sorgen und Schlaflosigkeit. Nur 15,6-46,1 \% (abhängig vom Kriterium) gaben an, dass ihre Ärzte diesen Problemen genug Aufmerksamkeit widmeten. Hier müsse laut Tauchert ein Umdenken stattfinden. Der Arzt müsse Themen wie sexuelle Störungen und soziale Probleme, Depressionen, Angstzustände, Sorgen und Schlaflosigkeit früher und aktiver mit seinen Patienten besprechen.

Doris Berger

Bericht von der Jahrestagung der European Society for Medical Oncology (ESMO) vom 28. September bis 2. Oktober 2012 in Wien. 